\title{
Crimean Congo Hemorrhagic Fever (CCHF) Review
}

\author{
Ajab Khan', Ghulam Abbas, ${ }^{2, *}$ Najeeb Ullah ${ }^{3}$, Syed Wsim Abbas ${ }^{4}$ \\ ${ }^{1}$ Department of Pathology, Faculty of Veterinary Sciences, University of Veterinary and Animal Sciences, Lahore, Pakistan \\ ${ }^{2}$ Department of Poultry Science, Riphah College of Veterinary Sciences, Lahore, Pakistan \\ ${ }^{3}$ Department of Plant Breeding and Genetics, Faculty of Crop and Food Sciences, \\ PMAS-Arid Agriculture University Rawalpindi, Pakistan \\ ${ }^{4}$ Department of Clinical Medicine and Surgery, University of Veterinary and Animal Sciences, Lahore, Pakistan
}

Copyright $\bigcirc 2018$ by authors, all rights reserved. Authors agree that this article remains permanently open access under the terms of the Creative Commons Attribution License 4.0 International License

\begin{abstract}
Crimean Congo hemorrhagic fever (CCHF) is a tick-borne disease caused by the arbovirus (a spherical enveloped RNA virus having diameter about $100 \mathrm{~nm})$. The virus belongs to Nairovirus genus of Bunyaviridae family. $\mathrm{CCHF}$ is a tick-borne viral disease of zoonotic importance prevalent in about 30 countries worldwide. Most susceptible species of virus are humans, livestock, mall vertebrates and ostriches. CCHF is also known as Congo fever, Central Asian hemorrhagic fever, Congo virus disease, Hungribta (blood taking), Crimean hemorrhagic fever, Khunymuny (nose bleeding), Karakhalak (black death), and viral tick-borne hemorrhagic fever disease. CCHF virus transmits to humans through tick biting (genus Hyalomma) and/or exposure to blood and/or tissues of infected livestock or direct contact with body fluids and/ or blood of viraemic persons. CCHF has been found fatal in humans but does not show clinical signs in Small vertebrates (excluding birds) and livestock. Most often mortality remains around $30 \%$, however it may range up to $5 \%$ to $80 \%$. Viremic person shows dramatic progression characterized by hemorrhages, chilling, sudden onset of high fever, headache, dizziness, gastrointestinal symptoms, severe back, and abdominal pain and myalgia, after 3-7 days of incubation. Other symptoms include diarrhea, vomiting, nausea, cardiovascular, and neuropsychiatric changes. However, in severe infection, hemorrhages ranging from petechiae to large areas of ecchymosis may develop. Early diagnosis using ELISA (enzyme-linked immunoassay) and molecular tools such as RT-PCR (real-time reverse transcription-polymerase chain reaction) is helpful for prevention and therapeutic measures. Although, currently there is no antiviral drug available for CCHF, however, Immunotherapy and Ribavirin have been found to be effective during sporadic outbreaks of disease. Further sound scientific research about basic biology of CCHFV may lead to the better treatment plan in the future.
\end{abstract}

Keywords CCHF, Arbovirus, Prevalent, Zoonosis, ELISA, RT-PCR

\section{Introduction}

Crimean-Congo hemorrhagic fever (CCHF) is the most important tick-borne viral disease, causing sporadic cases or outbreaks of severe illness across the globe. CCHFV is maintained in vertical and horizontal transmission cycles involving ixodid ticks in a variety of wild and domestic vertebrates, which do not show signs of illness. This virus is member of the genus Nairovirus, family Bunyaviridae and circulates in a number of tick genera, but Hyalomma ticks are the prime source of human infection, possibly because both juvenile and adult forms vigorously look for hosts for the blood meals required at each stage of maturation. CCHF occurs most commonly among agricultural workforce subsequent the bite of an infected tick and to a lesser degree amongst slaughterhouse staff exposed to the blood and tissues of infected livestock and medical staff through contact with the body fluids of infected patients $[10 ; 3]$. CCHF was first detected in the 1940's, high levels of genomic diversity argue against a recent origin [5]. It has been detected in at least 31 species of ticks in the Ixodidae (hard ticks) and Argasidae (soft ticks). These ticks have sexual, trans-ovarial (i.e., from infected mother to egg stage), and trans-stadial virus transmission i.e., from larva to nymph, from nymph to adult [18]. Pakistan is facing a quadruple burden of CCHF that needs extreme attention [24]. The first incident of CCHF in Pakistan was occurred in 1976; further fourteen cases were reported from 1976 to 2010 [25] Since then, an increase in the incidence of CCHF has been reported in the country [26]. Cattle herding is a regular practice in rural areas of the country whereas preventive and hygienic measures are rare due to poor infrastructure, scarcity of health associated resources and higher illiteracy rate in the village areas in the country. Usually these animals are then transported to urban areas for business purpose especially during Eid days, which can increase the threat of prevalence of the virus. In spite of repeated increase in prevalence of CCHF little attention is paid on the awareness campaign of CCHF. Therefore, the intent of this 
review is to provide general information about back ground, epidemiology, prevalence, prevention and treatment of CCHF.

\section{Background \& History}

Crimean-Congo hemorrhagic fever (CCHF) is a tick-borne disease caused by the arbovirus. CrimeanCongo hemorrhagic fever virus (CCHFV) is a member of the Nairovirus genus (family Bunyaviridae). CCHF was first recognized during a large outbreak among agricultural workers in the mid-1940 in the Crimean peninsula. A disease now considered to be CCHF was described by a physician in the $12^{\text {th }}$ century from the region that is presently Tadzhikistan. The description was of a hemorrhagic disease with the presence of blood in the urine, rectum, gums, vomitus, sputum, and abdominal cavity and was said to be caused by a louse or tick, which normally parasitizes a blackbird. The arthropod described may well have been a species of Hyalomma tick larvae which are frequently found on blackbirds. CCHF has also been recognized for centuries under at least three names by the indigenous people of southern Uzbekistan: khungribta(blood taking), khunymuny(nose bleeding), or karakhalak(Black Death)

As early as 1944, Hyalomma spp. ticks were implicated in the ecology of CCHF based upon a relationship between clinical cases and tick bite. In fact, the following year, a healthy volunteer subcutaneously inoculated with a suspension of 370 nymphal $H$. marginatumticks developed a disease characteristic of mild CCHF. This not only helped to prove the viral etiology of this disease, but also implicated Hyalomma spp. ticks as possible vectors; however, it was not until the late 1960's that the virus was isolated from adult Hyalomma, as well as several other tick species [7].

\section{Crimean-Congo Hemorrhagic Fever (CCHF)}

The disease now occurs sporadically throughout much of Africa, Asia, and Europe and results in an approximately $30 \%$ fatality rate. After a short incubation period, CCHF is characterized by a sudden onset of high fever, chills, severe headache, dizziness, back, and abdominal pains. Additional symptoms can include nausea, vomiting, diarrhea, neuropsychiatric, and cardiovascular changes. Numerous genera of ixodid ticks serve both as vector and reservoir for CCHFV; however, ticks in the genus Hyalomma are particularly important to the ecology of this virus. In fact, occurrence of CCHF closely approximates the known world distribution of Hyalomma spp. ticks. Therefore, exposure to these ticks represents a major risk factor for contracting disease. Like other tick-borne zoonotic agents, CCHFV generally circulates in nature unnoticed in an enzootic tick-vertebrate-tick cycle. Sera from several species of wild mammals have antibodies to
CCHFV and sero-epidemiological studies have also detected antibodies to CCHFV in domestic cattle, horses, donkeys, sheep, goats, and pigs from various parts of Europe, Asia, and Africa [13]. It is unclear whether the variation observed in incubation times, and ultimately disease outcome, may be due to this phenomenon or other factors, such as viral dose. After the incubation period, the pre hemorrhagic period is characterized by a sudden onset of fever, chills, severe headache, dizziness, photophobia, and back and abdominal pains. Additional symptoms such as nausea, vomiting, diarrhea and an accompanying loss of appetite are common. Fever is often very high $\left(39-41^{\circ} \mathrm{C}\right)$ and can be constantly elevated for 5-12 days or may be biphasic. It is interesting that neuropsychiatric changes have been reported in some CCHF patients. These have included sharp changes in mood, with feelings of confusion and aggression and even some bouts of violent behavior [16].

\section{Epidemiology}

From 2000 through 2008, the infection emerged or re-emerged in Bulgaria, Albania, Kosovo, and Turkey. It has also recently emerged in Greece, where the first human case has been recognized. This has been attributed to mild winters and to the disruption of agricultural activities, both accounting for an increased tick population, as well as to the migration or transportation of tick-infested birds or animals. Similarly to Kenya, Senegal, and South Africa, the 2002 outbreak in Northeastern Anatolia, Turkey, an eastern Black Sea extended geographic region, followed several decades of serologic evidence of a zoonotic focus, not preceded by any human case in the country. In 2007 and 2008, a considerable number of CCHF confirmed cases occurred in southeastern and western areas of Turkey, which in 2008 contributed $9 \%$ of cases. The infection has spread to previously non-endemic areas of Turkey, near the Aegean coast of the country and in a rural area of Ankara [11].

\section{Occurrence in Pakistan}

A nosocomial outbreak of Crimean-Congo hemorrhagic fever occurred in Rawalpindi, Pakistan in February 2002. The identified index case died shortly after admission to a hospital. Two of the health care workers became secondary cases; one of them died on day 13 after coming in contact with the index case. The other secondary case was successfully treated with oral ribavirin (Athar et al. 2003). CCHF was first reported in Pakistan in 1976 but the number of cases has shown a dramatic rise since 2000 with 50-60 cases being reported annually. The incidence of CCHF peaks in June and October but cases occur throughout the year. During 2012, DEWS received a total of $68 \mathrm{CCHF}$ alert involving 141 suspected and 49 
laboratory confirmed human cases resulting in 16 deaths (CFR: 33\%), while during 2013 up till 30th September a total of 150 human samples have been received by the NIH for $\mathrm{CCHF}$ testing. Out of them 60 are positive while among them 12 patients had died (CFR 8\%). On epidemiological analysis, the exposure of bulk of cases was traced back to animals in Quetta, Zhob, Killa Saifullah, Killa Abdullah, Pishin, Loralai and Musa Khel districts of Balochistan. Similarly a number of patients diagnosed in Karachi, Rawalpindi, Multan, D. I. Khan, Mansehra, Peshawar, Landi Kotal and recently from Abbottabad also possessed history of contact and slaughtering animals (NIH Islamabad 2013). From 1 January to 9 June 2013, a total of 16 suspected cases of Crimean-Congo haemorrhagic fever (CCHF), including six deaths (case-fatality rate $37.5 \%$ ) were reported from Pakistan. So far, 7 of these reported cases have been laboratory-confirmed. In 2012, the country faced a similar outbreak of CCHF with 61 suspected cases, including 17 deaths (case-fatality rate $27.8 \%$ ) reported from the disease. The majority of the cases were reported from the province of Balochistan, Sindh, Khyber Pakhtunkhwa and Punjab (WHO report 2013). Crimean-Congo virus, the causative agent of Crimean-Congo Virus Fever (CCVF) is endemic in Pakistan. Cases are documented sporadically ever year, mostly at and around the time of Eid-ul-Adha, an Islamic festival, celebrated on day 10 through 13 of the 12th month of each lunar calendar year. At this time of the year in Pakistan, livestock are brought down to the urban areas from the rural parts of the country. Animals are housed in open spaces and private houses until they are slaughtered during the 3 days of Eid-ul-Adha. This allows the CCHF virus, which is carried by a tick that inhabits the animal hide, to be transmitted through unprotected contact with live animals as well as through contact with animal blood subsequent to its slaughter. In this report, a typical case of CCVF is described that was encountered in Rawalpindi, Pakistan. A number of issues pertaining to the management of recurrent outbreaks of CCVF in the country are discussed [14]. Crimean-Congo hemorrhagic fever (CCHF) is endemic in certain rural areas of Pakistan. Since the discovery of CCHF virus (CCHFV) in the country in the 1960s, there have been 13 outbreaks in addition to sporadic cases. An outbreak during 2000 coincided with the movement of sacrificial animals from rural to urban areas for the festival of Eid-ul-Azha. Diagnosis was suspected in patients with fever and thrombocytopenia, and confirmed retrospectively using immunoassays and reverse transcriptase-PCR. Patients were given platelet, plasma and red cell infusions. Management varied due to unfamiliarity with the condition and its treatment, lack of availability of diagnostic laboratory tests and limited supply of ribavirin. Inadequate antiviral treatment and late presentation probably contributed to the death of six of the eight patients. Renal failure, disseminated intravascular coagulation and persistent high-grade fever were associated with mortality. The nucleotide sequence of the small genomic RNA segment of the CCHFV isolated in this outbreak was found to be very closely related to the CCHFV strains previously isolated in Pakistan [9]. Crimean - Congo hemorrhagic fever (CCHF) is a deadly viral disease that is endemic in some parts of Pakistan. We report here the first ever case of CCHF from Abbottabad. The patient presented with abdominal pain, hematemesis and low platelets, and died within 24 hours of admission. The diagnosis was confirmed by PCR for the CCHF virus. Because of the aggressive infection-control measures adopted, secondary and nosocomial spread was prevented [17].

Crimean-Congo hemorrhagic fever (CCHF) is endemic in the Baluchistan province, Pakistan. Sporadic outbreaks of CCHF occur throughout the year especially in individuals in contact with infected livestock. Nosocomial transmission remains a risk due to difficulties in the diagnosis of CCHF and limited availability of facilities for the isolation of suspected patients. Rapid diagnosis of CCHF virus infection is required for early management of the disease and to prevent transmission. This study describes the case of a 43-year-old surgeon who contracted CCHF during a surgical procedure in Quetta, Baluchistan and who was transferred to a tertiary care facility at the Aga Khan University Hospital, Karachi within 1 week of contracting the infection. Diagnosis of CCHF was made using a rapid real-time reverse transcription polymerase chain reaction (RT-PCR) assay for CCHF viral RNA. The patient had chronic hepatitis B and hepatitis D infection for which he had previously received a liver transplant. He proceeded to develop classic hemorrhagic manifestations and succumbed to the infection 14 days post-onset of disease. There was no further nosocomial transmission of the CCHF during the hospital treatment of the surgeon. Early diagnosis of CCHF enables rapid engagement of appropriate isolation, barrier nursing and infection control measures thus preventing nosocomial transmission of the virus [8].

Crimean - Congo hemorrhagic fever (CCHF) is a deadly viral disease that is endemic in some parts of Pakistan. We report here the first ever case of CCHF from Abbottabad. The patient presented with abdominal pain, hematemesis and low platelets, and died within 24 hours of admission. The diagnosis was confirmed by PCR for the CCHF virus. Because of the aggressive infection-control measures adopted, secondary and nosocomial spread was prevented [17]

Since 1976 to 2003, 14 epidemics, including 8 with nosocomial origin, have been reported in the country, mainly in the Western and North Western areas. In Balochistan province, the first sporadic case was documented in 1978, whereas recorded outbreaks have occurred yearly since 1987 and then in 1994, 1995, 1998, 2000 and 2001. According to National Institute of Health Islamabad, majority of IgM confirmed cases of CCHF 
found during 6 years (2003-2008) belong to Balochistan province (57), followed by Khyber Pakhtunkhwa (20), Punjab (6), Sindh (2). According to WHO, in September-2013, 4 deaths (2 confirmed for CCHF) reported from district Abbottabad, Khyber Pakhtunkhwa province, all 4 cases belonged to the same family living in Abbottabad, Khyber Pakhtunkhwa, and butchers by profession; while 4 more CCHF cases, 2 confirmed from district Killa Abdullah; and 2 suspected cases reported from districts Loralai and Musa Khail in Balochistan province were reported in 2013. So for a total of 72 suspected, 48 confirmed CCHF cases and 16 deaths have been reported country wide during the year 2013. In 2012, a total of 62 suspected cases were reported throughout the country with 41 cases confirmed and in total 18 deaths; of which 13 deaths (CFR is $31.7 \%$ ) were reported of the lab confirmed cases and 5 deaths were reported as suspected CCHF cases. 23 confirmed cases were reported from Balochistan, 7 from Sindh; 6 from Khyber Pakhtunkhwa and 5 from Punjab. Approximately all the cases had contact history with animal trading/handling, tick bite, contact with patient, tannery worker, butcher/animals slaughtering, and fresh animal skin handling. There is an ongoing trade of animals and animal skins with movement intra Pakistan and between neighboring countries i.e. Afghanistan and Iran (WHO report 2013).

Suspected cases of CCHF reported in Pakistan, 2000-2012

\begin{tabular}{|cccc|}
\hline Year & Cases & Deaths & Case-fatalityrate(\%) \\
\hline $\mathbf{2 0 0 0 - 2 0 0 2}$ & 191 & 59 & 26.2 \\
$\mathbf{2 0 0 3 - 2 0 0 6}$ & 328 & 42 & 12.8 \\
$\mathbf{2 0 1 0}$ & 29 & 3 & 4.9 \\
$\mathbf{2 0 1 2}$ & 61 & 17 & 27.8 \\
\hline
\end{tabular}

\section{Disease Vector}

Numerous genera of ixodid ticks serve both as vector and reservoir for CCHFV; however, ticks in the genus Hyalomma are particularly important to the ecology of this virus. In fact, occurrence of CCHF closely approximates the known world distribution of Hyalomma spp. ticks. Therefore, exposure to these ticks represents a major risk factor for contracting disease. This has been attributed to mild winters and to the disruption of agricultural activities, both accounting for an increased tick population, as well as to the migration or transportation of tick-infested birds or animals. Viral isolations from ticks have been made from two species in the family Argasidae (soft ticks) and from seven genera of the family Ixodidae (hard ticks). Far more important to the ecology and epidemiology of CCHF are ticks in the genus Hyalomma. In ticks that were experimentally infected with CCHFV, virus was recovered in highest titers from salivary glands and reproductive tissues and was positively associated with blood feeding. Additionally, virus was recovered from Malpighian tubules, mid gut, muscle, and nervous tissues from nearly all the ticks tested; however, viral titers were consistently low from these tissues. Although Hyalomma spp. Ticks are considered the most important in the epidemiology of CCHF; the virus has been isolated from ticks in other genera (i.e., Rhipicephalus, Boophilus, Dermacentor, and Ixodes spp.) as well, which may contribute to its wide geographical distribution. The biological role of ticks is also important, not only as virus vectors, but also as reservoirs of the virus in nature.

\section{Clinical Features}

Clinical symptoms of infection commonly include fever, myalgia, and hemorrhages. Levels of liver enzymes are raised, and bleeding markers are often increased. A role of inflammatory cytokines in the pathogenesis has been suggested, and CCHFV employs a range of passive and active mechanisms to avoid induction of the antiviral type I interferon [22].

The incubation period ranges from a few days up to 1 week, the length most probably depending on the transmission route and the amount of inoculums. The pre-hemorrhagic phase usually initiates with fever, myalgia, dizziness, headache and vomiting and ends on average after 3 days. The hemorrhagic fever phase is short and characterized by bleeding from the gastrointestinal system, urinary and respiratory tract and also skin bleeding ranging from petechiae to ecchymosis. Other symptoms include enlarged spleen and liver which approximately $30 \%$ of patients experience. The average mortality rate is $30 \%$ but can be as high as $70 \%$ [16].

The pathogenesis of CCHF is only poorly characterized due to several reasons such as (i) infections occur sporadically and in areas where facilities are limited for performing complete autopsies, (ii) virus handling requires biosafety level 4 (BSL-4) containment laboratories, and (iii) a lack of available animal models of disease. The limited knowledge about CCHF pathogenesis is mostly derived from blood analyses and liver biopsies of patients, using materials e.g. from small outbreaks in Turkey [2].The most comprehensive study involving $50 \mathrm{CCHF}$ patients from South Africa was undertaken by [16]. In their report, they describe that cerebral hemorrhage, severe anemia, severe dehydration, and shock associated with prolonged diarrhea, lung edema, and pleural effusion are the factors causing fatal outcome. Almost all patients who died developed multiple organ failure. In the fatal cases, platelet counts can be extremely from an early stage of illness.

\section{Diagnosis}

The fluorescence focus assay and the plaque assay in CER cells were compared with mouse inoculation for the isolation and titration of Crimean-Congo hemorrhagic 
fever virus. The fluorescence focus assay and the plaque assay were of similar sensitivity, but both produced 10- to 100 -fold lower titers than did mouse inoculation. For specimens from 26 Crimean-Congo hemorrhagic fever patients in South Africa, virus was isolated from 20 by mouse inoculation and from only 11 by cell culturing. Although cell cultures were less sensitive for the isolation of virus from clinical specimens, they produced diagnostic results much more rapidly [15].

We treated a male patient with Crimean-Congo hemorrhagic fever (CCHF). The diagnosis of CCHF was confirmed by reverse transcription-PCR and recombinant nucleoprotein (rNP)-based immunoglobulin G (IgG) and IgM capture enzyme-linked immunosorbent assays of serially collected serum samples. The patient was treated with intravenous ribavirin and recovered with no consequences. The study indicates that rNP-based CCHF virus antibody detection systems are useful for confirming CCHF virus infections. This case also suggests that intravenous ribavirin therapy may be promising for the treatment of CCHF patients [19]. Early and accurate diagnosis of Crimean-Congo hemorrhagic fever (CCHF) is essential for the treatment and outcome of the disease and prevention of its further transmission. Molecular-based diagnostic assays now serve as the front-line tool in the diagnosis of CCHF. However, the development of real-time RT-PCR assay for the detection of Crimean-Congo hemorrhagic fever virus (CCHFV) has been hampered by a virus strain variation. The development of a one-step real-time RT-PCR assay for the detection of CCHFV is described herein. The technique is based on the fluorescence resonance energy transfer probe technology employing the endonuclease activity of Taq polymerase enzyme. The assay was designed to detect specifically the strains from a phylogenetic cluster of CCHFV which encompasses the known CCHFV strains circulating in the Balkan region. The detection system was tested using CCHFV strain Kosovo Hoti, clinical serum samples and ticks. The real-time assay described is rapid, specific and sensitive. Since the Balkan peninsula is also an endemic region for hemorrhagic fever with renal syndrome (HFRS), this method is suggested as convenient for early differential diagnosis of suspected viral hemorrhagic fever patients [6].

\section{Virus Isolation}

Any attempts at isolating and culturing the virus should only be performed in a maximum bio-containment laboratory (i.e., BSL-4). The traditional method for CCHFV isolation has been by intracranial (i.e.) or intra peritoneal (i.p.) inoculation of a sample (e.g., blood from an acute-phase patient or ground tick pools) into newborn mice. Isolation in cell culture is far simpler and provides a more rapid result, but is generally considered less sensitive.
Depending on the cell line and strain, the virus may produce little or no cytopathic effect (CPE) and develop into a non-cytopathic persistent infection of the cells; however, virus can be identified by performing immunofluorescence assay (IFA) with specific monoclonal antibodies [16].

\section{Treatment}

Treatment options for CCHF are limited. Immunotherapy and ribavirin have been tried with varying degrees of success during sporadic outbreaks of disease, but no case-controlled trials have been conducted. Consequently, there is currently no antiviral treatment for CCHF approved by the U.S. Food and Drug Administration (FDA). However, renewed interested in CCHFV, as well as increased knowledge of its basic biology, may lead to improved therapies in the future [21].

\section{Prevention and Control}

\section{Risk Factors}

There are several groups of individuals who are considered to be at-risk of contracting CCHFV. Specifically, people from endemic areas who are susceptible to tick bite, particularly from Hyalomma spp. ticks. These would include individuals who work outdoors, particularly those who work with large domestic animals. Although CCHFV has been isolated from numerous species of ticks those of the Hyalomma genus are considered the primary vector in CCHF enzootic and endemic areas. The distribution of CCHFV coincides precisely with the distribution of Hyalomma ticks, therefore, there appears to be little or no risk in areas outside the known distribution of these ticks. Exposures such as crushing infected ticks and butchering infected animals have also been a frequent source of CCHFV infection. Other groups who are at-risk include those caring for CCHF patients. In fact, the risk of nosocomial infection in health-care workers is well documented and can be extremely high, especially during the hemorrhagic period of disease [12]. This is exemplified by a nosocomial case that reported in the Central Government Hospital in Pakistan in January 1976. A shepherd was brought to the hospital with typical CCHF symptoms and died the same night. The shepherd's father, who cared for his sick son at home, was hospitalized and died 2 days later despite intensive care and repeated blood transfusions. A female physician, who admitted the shepherd, when he vomited blood onto her face and hands, showed signs of CCHFV infection and was hospitalized, but ultimately recovered. The boy had surgery on the day of his hospital admission. The surgeon, who cut his finger while operating, died of CCHF 2 weeks later. An assistant surgeon who pricked his finger during the operation also contracted CCHF and was 
hospitalized; he later recovered. A nursing attendant who assisted in the operation died of CCHF 3 days later. The anesthesiologist also became ill and experienced bleeding from the gums, but recovered. Five of seven other persons in the operating theatre during the procedure also became ill and were hospitalized; all recovered. Of the 12 hospital personnel attending the shepherd, 10 became ill with CCHF; two died and eight recovered after severe illness. Another nosocomial outbreak occurred at Tygerberg Hospital in South Africa. Thirty-three percent of medical workers who had contact with patients through accidental needle prick developed CCHF and $8.7 \%$ contracted disease by other contacts with the patients' blood [20].

\section{Control Measures}

The best means of preventing disease is to avoid or minimize exposure to the virus. This can be accomplished in a number of ways. Persons in high-risk occupations (i.e. Slaughterhouse workers, veterinarians, sheep herders, etc.) should take every precaution to avoid exposure to virus infected ticks or virus-contaminated animal blood or other tissues. For example, wearing gloves and limiting exposure of naked skin to fresh blood and other tissues of animals are effective practical control measures. Likewise, medical personnel who care for suspected CCHF patients should practice standard barrier-nursing techniques. Acaricide treatment of livestock in CCHFV endemic areas is effective in reducing the population of infected ticks.

\section{Potential Bioterrorism Concerns}

The highly pathogenic nature of the CCHFV has led to the fear that it might be used as an agent of bioterrorism and/or bio warfare and has resulted in its inclusion as a CDC/NIAID Category C Priority Pathogen. CCHFV can be transmitted from person to person, has a high case-fatality rate, and may be transmissible by small-particle aerosol; but, its inability to replicate to high concentrations in cell culture is cited as a major impediment to its development as a mass casualty weapon (Borio et al. 2002) and thus precludes its classification as a Category A or B pathogen. The highly lethal nature of the virus has restricted research to BSL-4 laboratories and has consequently had limited research investigations.

\section{Public Health Hazzards}

CCHF cases occurring as an expected event in endemic areas should be notified to clinicians in the international neighborhood. They should be aware of the probability of importation of CCHF cases from endemic areas, of human-to-human transmission, particularly in the nosocomial setting, and of the potential transmission of the virus via tick-infested and infected imported livestock.
This novel European CCHF geographic distribution is a challenge for the scientific community of medical microbiologists, epidemiologists, medical entomologists, and veterinarians that could be followed by acceleration of a European Standardized Response at the national, regional, and international level [20].

Humans become infected with CCHF virus either through bites of infected ticks, which maintain a life-long infection and are competent reservoirs, or by direct contact with virus contaminated tissues or blood. In addition to zoonotic transmission, CCHF virus can spread from person to person and is one of the rare haemorrhagic fever viruses able to cause nosocomial outbreaks in hospitals with high standards of hygiene [6]. Human beings are the only known hosts of CCHF virus, in which disease is manifested as an acute febrile illness followed by a fatal haemorrhagic syndrome with mortality rates of up to $50 \%$ [15]. Shepherds, campers, agricultural workers, veterinarians, abattoir workers, and other persons in close contact with livestock and ticks are at higher risk of infection [23].

\section{REFERENCES}

[1] Athar MN, Baqai HZ, Ahmad M, Khalid MA, Bashir N, Ahmad AM, Balouch AH, Bashir K. 2003. Short report: crimean-congo hemorrhagic fever outbreak in Rawalpindi, Pakistan, February 2002. The American journal of tropical medicine and hygiene. 69 (3): 284-287.

[2] Akıncı E, Yılmaz M, Bodur H, Öngürü P, Bayazıt FN, Erbay A, Özet G. 2009. Analysis of lymphocyte subgroups in Crimean-Congo hemorrhagic fever. International Journal of Infectious Diseases. 13 (5): 560-563.

[3] Bente DA, Forrester NL, Watts DM, McAuley AJ, Whitehouse CA, Bray M. 2013. Crimean-Congo hemorrhagic fever: History, epidemiology, pathogenesis, clinical syndrome and genetic diversity. Antiviral research. 100 (1): 159-189.

[4] Borio L, Inglesby T, Peters C, Schmaljohn AL, Hughes JM, Jahrling PB, Ksiazek T, Johnson KM, Meyerhoff A, O'Toole T. 2002. Hemorrhagic fever viruses as biological weapons: medical and public health management. Jama. 287 (18): 2391-2405.

[5] Carroll SA, Bird BH, Rollin PE, Nichol ST. 2010. Ancient common ancestry of Crimean-Congo hemorrhagic fever virus. Molecular Phylogenetics and Evolution. 55 (3): 1103-1110.Crimean-Congo haemorrhagic fever (CCHF) in Pakistan, 14 June 2013.

[6] Duh D, Saksida A, Petrovec M, Dedushaj I, Avšič-Županc T. 2006. Novel one-step real-time RT-PCR assay for rapid and specific diagnosis of Crimean-Congo hemorrhagic fever encountered in the Balkans. Journal of virological methods. 133 (2): 175-179.

[7] Hoogstraal H 1979. The epidemiology of tick-borne Crimean-Congo hemorrhagic fever in Asia, Europe, and Africa. Journal of medical entomology. 15 (4): 307-417. 
[8] Hasan Z, Mahmood F, Jamil B, Atkinson B, Mohammed M, Samreen A, Altaf L, Moatter T, Hewson R. 2013. CrimeanCongo hemorrhagic fever nosocomial infection in a immunosuppressed patient, Pakistan: Case report and virological investigation. Journal of medical virology. 85 (3): 501-504.

[9] Jamil B, Hasan RS, Sarwari AR, Burton J, Hewson R, Clegg C. 2005. Crimean-Congo hemorrhagic fever: experience at a tertiary care hospital in Karachi, Pakistan. Transactions of the Royal Society of Tropical Medicine and Hygiene. 99 (8): 577-584.

[10] Mehravaran A, Moradi M, Telmadarraiy Z, Mostafavi E, Moradi AR, Khakifirouz S, Shah-Hosseini N, Varaie FSR, Jalali T, Hekmat S. 2012. Ticks and Tick-borne Diseases.

[11] Nabeth P, Thior M, Faye O, Simon F. 2004. Human Crimean-Congo hemorrhagic fever, Senegal. Emerging infectious diseases. 10 (10): 1881.

[12] Papa A, Bino S, Llagami A, Brahimaj B, Papadimitriou E, Pavlidou V, Velo E, Cahani G, Hajdini M, Pilaca A. 2002. Crimean-Congo hemorrhagic fever in Albania, 2001. European Journal of Clinical Microbiology and Infectious Diseases. 21 (8): 603-606.

[13] Paragas J, Whitehouse CA, Endy TP, Bray M. 2004. A simple assay for determining antiviral activity against Crimean-Congo hemorrhagic fever virus. Antiviral research. 62 (1): $21-25$.

[14] Rai MA, Khanani MR, Warraich HJ, Hayat A, Ali SH. 2008. Crimean - Congo hemorrhagic fever in Pakistan. Journal of medical virology. 80 (6): 1004-1006.

[15] Shepherd A, Swanepoel R, Leman P, Shepherd S. 1986. Comparison of methods for isolation and titration of Crimean-Congo hemorrhagic fever virus. Journal of clinical microbiology. 24 (4): 654-656.

[16] Swanepoel R, Gill D, Shepherd A, Leman P, Mynhardt J, Harvey S. 1989. The clinical pathology of Crimean-Congo hemorrhagic fever. Review of Infectious Diseases. 11 (Supplement 4): S794-S800.

[17] Saleem J, Usman M, Nadeem A, Sethi SA, Salman M. 2009.
Crimean-Congo hemorrhagic fever: a first case from Abbottabad, Pakistan. International Journal of Infectious Diseases. 13 (3): e121-e123.

[18] Tahmasebi F, Ghiasi S, Mostafavi E, Moradi M, Piazak N, Mozafari A, Haeri A, Fooks A, Chinikar S. 2010. Molecular epidemiology of crimean-congo hemorrhagic fever virus genome isolated of ticks from Hamadan province of Iran.

[19] Tang Q, Saijo M, Zhang Y, Asiguma M, Tianshu D, Han L, Shimayi B, Maeda A, Kurane I, Morikawa S. 2003. A patient with Crimean-Congo hemorrhagic fever serologically diagnosed by recombinant nucleoprotein-based antibody detection systems. Clinical and diagnostic laboratory immunology. 10 (3): 489-491.

[20] Van De Wal B, Joubert J, Van Eeden P, King J. 1985. A nosocomial outbreak of Crimean-Congo haemorrhagic fever at Tygerberg Hospital. Part IV. Preventive and prophylactic measures.

[21] Whitehouse CA 2004. Crimean-Congo hemorrhagic fever. Antiviral research. 64 (3): 145-160.

[22] Weber F, Mirazimi A. 2008. Interferon and cytokine responses to Crimean Congo hemorrhagic fever virus; an emerging and neglected viral zonoosis. Cytokine \& growth factor reviews. 19 (5): 395-404.

[23] Zakhashvili K, Tsertsvadze N, Chikviladze T, Jghenti E, Bekaia M, Kuchuloria T, Hepburn MJ, Imnadze $P$, Nanuashvili A. 2010. Crimean-Congo hemorrhagic fever in man, Republic of Georgia, 2009. Emerging infectious diseases. 16 (8): 1326.

[24] Haider, S., M. A. Hassali, Q. Iqbal, M. Anwer, F. Saleem. 2016. Crimean-Congo haemorrhagic fever in Pakistan. The Lancet Infectious Disease, 16 (12): 1333.

[25] Qidwai, W. Crimean-Congo haemorrhagic fever: an emerging public health care challenge in Pakistan. J Coll Physicians Surg Pak. 2016; 26: 81-82.

[26] WHO. Crimean-Congo haemorrhagic fever in Pakistan. ((accessed Sept 20, 2016).) http://www.emro.who.int/surveillance-forecasting-respons e/surveillance-news/cchf-july-2014.html. Date: 2016 